\title{
BMR
}

\section{Relationships between transient elastography values and liver fibrosis in chronic liver disease patients with normal or mildly abnormal aminotransferase levels}

\author{
Y.P. Zhang ${ }^{1 *}$, Q. Zhao ${ }^{1 *}$, Y.Z. Tao ${ }^{1}$, X.R. Niu $^{2}$ and L. Rui ${ }^{1}$ \\ ${ }^{1}$ Liver Disease Center, \\ The People's Hospital of Xinjiang Uygur Autonomous Region, China \\ ${ }^{2}$ Surgical ICU, \\ The People's Hospital of Xinjiang Uygur Autonomous Region, China \\ *These authors contributed equally to this study. \\ Corresponding author: L. Rui \\ E-mail: xinhosptial@163.com
}

Genet. Mol. Res. 14 (4): 18172-18180 (2015)

Received November 13, 2014

Accepted February 23, 2015

Published December 23, 2015

DOI http://dx.doi.org/10.4238/2015.December.23.4

\begin{abstract}
This study aimed to evaluate relationships between transient elastography values and liver fibrosis in chronic liver disease patients with normal or mildly abnormal aminotransferase levels. Fiftysix patients were enrolled in the study. Transient elastography and liver biopsy were performed on the same day, and the fibrosis was staged based on the Scheuer scoring system. Liver stiffness was measured to assessed liver fibrosis using transient elastography. The transient elastography values of 12 patients with chronic hepatitis B were studied before and 6 months after antiviral treatment. The sensitivity and specificity for $10.88 \mathrm{kPa}$ in S3 were 80 and $87.8 \%$, and for $19.4 \mathrm{kPa}$ in S4, were 100 and $90.7 \%$, respectively. In univariate analysis, liver stiffness strongly correlated with the fibrosis stage $(\mathrm{r}=0.70, \mathrm{P}<0.5)$, moderately correlated with the aminotransferases $(r=0.398, \mathrm{P}<0.05)$,
\end{abstract}


and poorly correlated with the degree of necroinflammatory activity ( $\mathrm{r}$ $=0.19, \mathrm{P}<0.5)$. In multivariate regression, liver stiffness correlated only with the fibrosis stage $(\mathrm{P}<0.05)$. Pre- and post-treatment viral loads were not significantly different $\left[(4.81 \pm 0.15) \times 10^{6} v s(7.62 \pm 0\right.$. 16) $\left.\mathrm{x} 10^{3}, \mathrm{P}>0.05\right]$. Pre- and post-treatment LS measurements were not correlated with viral load $(\mathrm{P}>0.05)$. Pre- and post-treatment LS measurements were not significantly different $(\mathrm{P}>0.02)$. In conclusion, transient elastography values correlated with the stage of cirrhosis, alanine aminotransferase levels, and antiviral treatment in patients with chronic hepatitis B and did not correlate with viral loads.

Key words: Chronic liver disease; Liver biopsy; Liver fibrosis; Transient elastography

\section{INTRODUCTION}

Chronic liver disease (CLD) is common worldwide. Chronic viral infections (B, C or $\mathrm{B}+\mathrm{C}$ ), alcohol, medications, autoimmune conditions, and non-alcoholic fatty liver disease (NAFLD) comprise the primary etiological factors. Some CLD patients with normal or mildly abnormal alanine aminotransferase (ALT) levels have no clinical symptoms and do not seek medical attention even when the disease progresses to cause gastrointestinal bleeding and/or ascites. Early detection of liver fibrosis and cirrhosis is important for prevention of disease progression by treatment with traditional Chinese medicines. Liver biopsy (LB) has been regarded as the gold standard for early detection of liver fibrosis and cirrhosis. However, LB is often limited by its invasiveness, sampling error, and intra/inter-observer variability in histological interpretation. Furthermore, it is not practical to repeat LB examinations within short time intervals (Ferraioli et al., 2013). Transient elastography (TE) is a novel ultrasound-based technology that allows a non-invasive measurement of liver stiffness (LS). TE is performed with a FibroScan ${ }^{\mathrm{TM}}$ (FS) device (Echosens, France), which incorporates a 5-MHz ultrasound transducer probe mounted on the axis of a vibrator. The vibrator generates a painless vibration, which in turn generates an elastic shear wave that propagates through the skin and the subcutaneous tissue into the liver. The shear wave velocity expressed in $\mathrm{kPa}$ is directly related to the stiffness of the tissue. The harder the tissue, the faster the shear waves propagate (Sandrin et al., 2003). TE allows evaluation of the elasticity of a portion of liver parenchyma that is approximately 100 times greater than that examined by biopsy (Castera et al., 2008). Recently, FS has become increasingly popular because of its non-invasiveness, convenience, high accuracy, good reproducibility, and accurate quantitative results. Several studies have shown significant positive correlations between TE and the stages of liver fibrosis (Foucher et al., 2006; Takeda et al., 2006; Ganne-Carriéet al., 2006; Carrión et al., 2006; Castera, 2008; Fraquelli et al., 2011). TE is most suitable in advanced stages of fibrosis (de Lédinghen et al., 2006; Kazemi et al., 2006; Vizzutti et al., 2007; Fraquelli et al., 2007; Arena et al., 2008; Rockey, 2008; Castera, 2011). A limitation of all the studies performed thus far is that the reference shear wave TE values for each fibrosis stage were calculated from their own cohort of patients after data collection, and these differ across studies (Shaheen et al., 2007; de Ledinghen and Vergniol, 2008; Stebbing et al., 2010; Tsochatzis et al., 2011). The studies mainly focused on chronic hepatitis $\mathrm{C} / \mathrm{B}$, with special emphasis on chronic hepatitis $\mathrm{C}$ patients (Ziol 
et al., 2005; Rigamonti et al., 2008; Lemoine et al., 2008; Chen et al., 2012; de Lédinghen et al., 2013; Feier et al., 2013; Calvaruso et al., 2013). The shear wave values of 7, 9.5, and 12 $\mathrm{kPa}$ corresponding to stages F2, F3, and F4, respectively, were used. In our hospital, the shear wave values of $5,7.3,9.7,12.4$, and $17.5 \mathrm{kPa}$ corresponding to stages F0, F1, F2, F3, and F4, respectively, were provided by the manufacturer. The aim of the current study was to validate these shear wave values by a comparison between these and LB results or obtain better shear wave values. In addition, we aimed to determine if the TE values changed during follow-up of hepatitis B virus (HBV) patients treated with antiviral agents.

\section{MATERIAL AND METHODS}

\section{Patients}

\section{Inclusion criteria}

From June 2011 through February 2013, 56 asymptomatic patients with CLD with normal or mildly abnormal ALT levels and scheduled for LB at the Liver Diseases Department of the People's Hospital of Xinjiang Uygur Autonomous Region, China, were enrolled in the study. The CLD etiology was determined using standard diagnostic criteria (Ziol et al., 2005). Patients were diagnosed with hepatitis $\mathrm{C}$ virus (HCV) or HBV based on serological detection of hepatitis $\mathrm{C}$ antibodies (with positive serum HCV RNA) by polymerase chain reaction or hepatitis B surface antigen with normal or mildly abnormal ALT levels over 6 months, respectively. Patient characteristics, epidemiological data, and biochemical test results were recorded. LB was performed on the same day as TE.

\section{Exclusion criteria}

Patients with symptomatic CLD, ALT $\geq 200 \mathrm{U} / \mathrm{L}$, or no LB were excluded. The normal ranges were ALT, $0-40 \mathrm{U} / \mathrm{L}$; platelet count, $10-30 \times 10^{3} / \mu \mathrm{L}$; serum albumin, $35-55 \mathrm{~g} / \mathrm{dL}$; and $\mathrm{HBV}$ DNA $<500 \mathrm{IU} / \mathrm{mL}$.

\section{Ethics statement}

The study protocol was approved by the Ethics Committee of the People's Hospital of Xin Jiang Uygur Autonomous Region. All participants provided written informed consent for study participation.

\section{TE}

TE was carried out using $\mathrm{FS}^{\mathrm{TM}}$ for measurements of LS at the right 7-8 or 8-9 intercostal spaces, with patients in the dorsal decubitus position and their right arm in maximal abduction. Only patients with 10 validated measurements, a success rate of at least $60 \%$, and interquartile range of less than $30 \%$ of the median LS value were included in the study. Values were expressed in $\mathrm{kPa}$.

The shear wave values of 5, 7.3, 9.7, 12.4, and $17.5 \mathrm{kPa}$ were used for stages F0, F1, F2, F3, and F4, respectively. F0, no fibrosis, corresponded to S0; F1, mild fibrosis, corresponded 
to $\mathrm{S} 1 ; \mathrm{F} 2$, moderate fibrosis, corresponded to S2; F3, severe fibrosis, corresponded to S3; and F4, cirrhosis, corresponded to S4. LS values over $17.5 \mathrm{kPa}$ were considered to be stage F4.

\section{Liver biopsy and histology}

Ultrasound-assisted percutaneous LB was performed using an intercostal approach and disposable $1.6 \mathrm{~mm}$-diameter modified MAGNUM needles (Montebell BARD, USA). All biopsy specimens were fixed in formalin and embedded in paraffin and stained with hematoxylin-eosin and reticulin and masson's trichome stain . The length $(\mathrm{mm})$ of each LB specimen was recorded. The specimens were read on site by a single expert liver pathologist who was blind to the TE results but not to the patient's clinical and biochemical data. Fibrosis was evaluated semiquantitatively and divided into 5 levels according to the degree of fibrosis, using the Scheuer scoring system (S0, absent; S1, enlarged fibrotic portal tract; S2, periportal or initial portal septa, but intact architecture; S3, architectural distortion, but no obvious cirrhosis; and S4, cirrhosis). Necroinflammatory activity was evaluated semi-quantitatively and also divided into 5 levels according to the degree of inflammation in the portal tract and lobe (G0, absent in the portal tract and lobe; G1, absent in the portal tract, degeneration, a little spotty necrosis, and focal necrosis of the lobe; G2, mild piecemeal necrosis in the portal tract, spotty necrosis, focal necrosis, and eosinophilic bodies in the lobules; G3, moderate piecemeal necrosis in the portal tract, degeneration, confluent necrosis, and/or bridging necrosis in the lobules; G4, severe piecemeal necrosis in the portal tract, enlarged bridging necrosis, and multilobular necrosis).

\section{Statistical analysis}

The statistical analysis was carried out using the SPSS software, version 16.0 (SPSS, Inc., Chicago, IL, USA). The distribution of numerical variables was first tested by the Kolmogorov-Smirnov test. In the case of numerical variables with a normal distribution, the mean value and standard deviation were calculated. Qualitative variables were presented as numbers and percentages. The diagnostic performance of TE for predicting significant liver fibrosis was assessed using the area under receiver operating characteristic (AUROC) curves. Optimal shear wave values were chosen to maximize the sum of sensitivity (Se) and specificity (Sp). For each predictive test, $95 \%$ confidence intervals (CIs) were calculated. Linear regression models of LS value, and ALT, platelet counts, and serum albumin levels were evaluated. P < 0.05 was considered to be statistically significant.

\section{Follow-up}

Twelve patients with chronic hepatitis B received antiviral treatment. Liver stiffness measurement LSM and HBV DNA values before treatment and 6 months after treatment were compared.

\section{RESULTS}

\section{Patients}

Fifty-six patients (34 men, 22 women) met the inclusion criteria, and their character- 
istics are summarized in Table 1. Forty-two (75\%) patients had chronic hepatitis B, $3(5.36 \%)$ had chronic hepatitis C, and 1 (1.79\%) was co-infected with hepatitis B and C; 2 (3.57\%) patients had NAFLD, $6(10.71 \%)$ had autoimmune hepatic disease, and $2(3.57 \%)$ had no definite diagnosis. LS measurements ranged from 2.5 to $39.7 \mathrm{kPa}$, as shown in Table 1 .

\begin{tabular}{lc} 
Table 1. Baseline characteristics of the patients. \\
\hline Major clinic indexes (N=56) \\
\hline Age (years) \\
Gender \\
Male & $41.52 \pm 9.95(16-68)$ \\
Female & $\mathrm{N}=34$ \\
Etiology & $\mathrm{N}=22$ \\
Viral (B/C/B+C) & $\mathrm{N}=42 / 3 / 1(75 / 5.36 / 1.79 \%)$ \\
NAFLD & $\mathrm{N}=2(3.57 \%)$ \\
Autoimmune & $\mathrm{N}=6(10.71 \%)$ \\
Other & $\mathrm{N}=2(3.57 \%)$ \\
Parameter & \\
ALT (IU/L) & $49.64 \pm 31.46$ \\
Platelet $\left(10^{3} / \mu \mathrm{L}\right)$ & $197.61 \pm 53.105$ \\
Albumin $(\mathrm{g} / \mathrm{dL})$ & $4.23 \pm 0.80$ \\
Scheuer scoring system & \\
Stage of fibrosis & \\
S0/S1/S2/S3/S4 & $\mathrm{N}=4 / 26 / 9 / 15 / 2$ \\
Grade of inflammation & $\mathrm{N}=1 / 15 / 24 / 14 / 2$ \\
G0/G1/G2/G3/G4 & \\
Company provided cut-off value (kPa) & $\mathrm{N}=15$ \\
F0 $<5.0$ & $\mathrm{~N}=14$ \\
$5.0<\mathrm{F} 1 \leq 7.3$ & $\mathrm{~N}=10$ \\
$7.3<\mathrm{F} 2 \leq 9.7$ & $\mathrm{~N}=1$ \\
$9.7<\mathrm{F} 3 \leq 12.4$ & $\mathrm{~N}=16$ \\
F4 $>12.4$ & $\mathrm{~N}=12$ \\
Follow-up & \\
Viral load (IU/mL) & \\
Viral load before treatment & $(4.81 \pm 0.15) \times 10^{6}$ \\
Viral load after 6 months of treatment & $(7.62 \pm 0.16) \times 10^{3}$ \\
LS measurements before treatment & $11.958 \pm 9.32$ \\
LS measurements after 6 months of treatment & $11.067 \pm 10.25$ \\
\hline &
\end{tabular}

\section{Relationship between LS and histology}

Patient distribution according to the fibrosis grade was as follows: S0, 4; S1, 26; S2, 9; $\mathrm{S} 3,15$; and $\mathrm{S} 4,2$. Comparing the $\mathrm{S} 0, \mathrm{~S} 1$, and $\mathrm{S} 2$ grades to the LB results, the area under the curve (AUC) was $0.195,0.21$, and 0.511 , respectively. The $95 \%$ CIs were $0.32-0.421,0.097$ 0.345 , and $0.342-0.679$, respectively. There was no diagnostic value in detecting liver fibrosis. With grade S3, the AUC was 0.875 , and $95 \% \mathrm{CI}$ was $0.769-0.981$. The diagnostic value was moderate. The LS measurements range was $5.1-39.7 \mathrm{kPa}$. When the shear wave value was $10.88 \mathrm{kPa}$, the Se was $80 \%$, and the Sp was $87.8 \%$ (Figure 1A). When the grade was S4, the LS measurement range was $21.1-27.4 \mathrm{kPa}$, the AUC was 0.926 , and the $95 \% \mathrm{CI}$ was $0.854-$ 0.998 . Here, the diagnostic value was the highest for detection of cirrhosis. When the shear wave value was $19.4 \mathrm{KPa}$, the Se was $100 \%$, and the Sp was $90.7 \%$, as shown in Table 2 . The ROC curve analysis identified optimal shear wave values of LS measurements as high as $10.88 \mathrm{kPa}$ for $\mathrm{S}=3$, and $19.45 \mathrm{kPa}$ for $\mathrm{S}=4$ (Figure 1B). Corresponding values of S3, S4 positive predictive value, negative predictive value, and AUCs are shown in Table 2. 
A
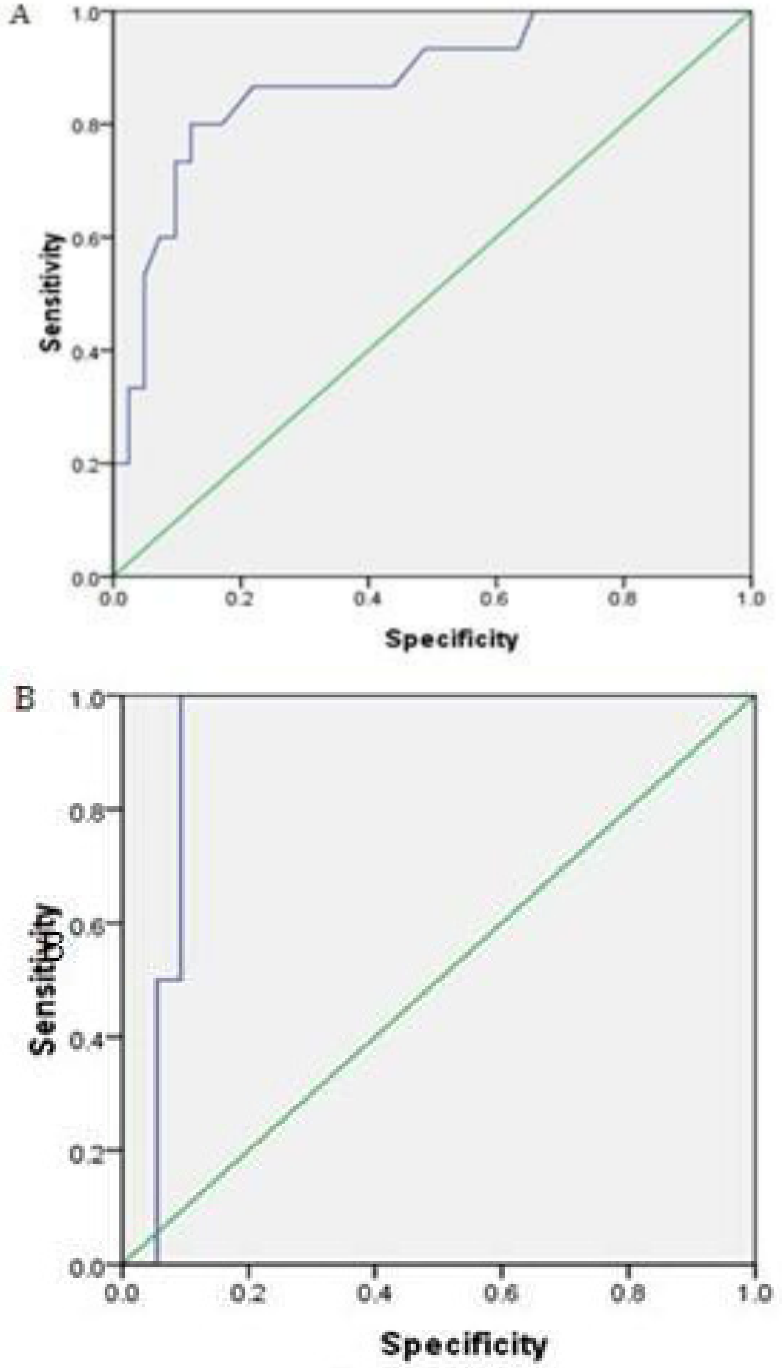

Figure 1. ROC curve analysis. A. Cut-off value: $10.88 \mathrm{kPa}$, AUC: 0.875 (95\%CI $=0.769-0.981)$ Sensitivity: 80\%, Specificity: 87.8\%. B. Cut-off value: $19.45 \mathrm{kPa}$, AUC: 0.926 (95\%CI = 0.854-0.998). Sensitivity: 100\%, Specificity: $90.7 \%$.

Table 2. Cut-off values of transient elastography obtained by receiver operating characteristic curve analysis for each fibrosis stage.

\begin{tabular}{lcccrr}
\hline LB & LSV $(\mathrm{kPa})$ & Sensitivity $(\%)$ & Specificity $(\%)$ & AUC & 95\%CI \\
\hline S0 & 4.95 & 50 & 23.1 & 0.195 & $-0.32-0.421$ \\
S1 & 5.65 & 50 & 20 & 0.221 & $0.097-0.345$ \\
S2 & 5.95 & 77.8 & 40.4 & 0.511 & $0.342-0.679$ \\
S3 & 10.88 & 80 & 87.8 & 0.875 & $0.769-0.981$ \\
S4 & 19.45 & 100 & 90.7 & 0.926 & $0.854-0.998$ \\
\hline
\end{tabular}


In univariate analysis, TE showed a good correlation with the degree of fibrosis $(\mathrm{r}=$ $0.700, \mathrm{P}=0.00)$, a moderate correlation with the ALT value $(\mathrm{r}=0.398, \mathrm{P}=0.002)$, and poor correlation with the degree of necroinflammation despite being statistically significant $(\mathrm{r}=$ $0.19, \mathrm{P}=0.002)$. LS also showed a negative correlation with serum albumin levels $(\mathrm{r}=-2.97$, $\mathrm{P}=0.026)$ and platelet count $(\mathrm{r}=-0.05, \mathrm{P}=0.715)$. Multivariate regression analysis including fibrosis stage, aspartate aminotransferase, and platelet counts confirmed the correlation of TE only with the fibrosis stage $(\mathrm{P}=0.000)$, but not with any other variables.

\section{LS between LB (S0-4) and standard (F0-4) provided by the manufacturer}

The number of patients with S0 was 4, and the number with F0 was 15 . Therefore, 11 patients may have been mistakenly diagnosed as F0. The number of patients with S1 was 26, and with F1 was 14 . Therefore, 12 patients may have been mistakenly diagnosed as F1. The number of patients with S2 was 9, and with F2 was 10. Here, 1 patient may have been mistakenly diagnosed as F2. Similarly, the number of patients with S3 was 15, and with F3 was 1. This indicates that 14 patients may have been mistakenly diagnosed as F3. The number of patients with S4 was 2, and with F4 was 16 . Therefore, 14 patients may have been mistakenly diagnosed as F4.

\section{Relationship of LS to virological response and pre- and post-treatment}

Twelve of 56 patients with chronic hepatitis B were treated. Before treatment, the mean of the viral load was $(4.81 \pm 0.15) \times 10^{6} \mathrm{IU} / \mathrm{mL}$, and the mean LS measurement was $11.96 \pm 9.32 \mathrm{kPa}$. Six months after treatment, the viral load was $(7.62 \pm 0.16) \times 10^{3} \mathrm{IU} / \mathrm{mL}$ and the mean LS measurement was $11.07 \pm 10.25 \mathrm{kPa}$. Pre- and post-treatment viral loads were not significantly different $(\mathrm{P}=0.785)$. Pre- and post-treatment LS measurements were not correlated with viral load $(\mathrm{P}=0.409)$. Pre- and post-treatment LS measurements were not significantly different $(\mathrm{P}=0.695)$. LS measurements decreased in 7 patients, did not change in 2 , and increased in 5 .

\section{DISCUSSION}

Liver fibrosis involves a pathological process commonly caused by various CLD. Progression of liver fibrosis can lead to cirrhosis. Therefore, it is important to accurately determine the stage of fibrosis for clinical diagnosis and treatment. The diagnostic LS value of S1-2 was very low for mild to moderate fibrosis. For diagnosis of fibrosis $\geq S 3$, the shear wave values were in the range of 9.5-12.0 $\mathrm{kPa}$ (Tsochatzis et al., 2011). In the current study, this shear wave value was $10.8 \mathrm{kPa}$. For a diagnosis of cirrhosis, the shear wave values were in the range of 12.4-17.6 $\mathrm{kPa}$ (Ziol et al., 2005; Castéra et al., 2005). In a previous study (Foucher et al., 2006), the shear wave value for a diagnosis of cirrhosis was $19.45 \mathrm{kPa}$. These differences could be due to fact that the study population included patients with CLD of various etiologies. In the previous studies, only patients with chronic HCV and HBV infections were included. The other reason for these differences could be a small sample size.

In univariate analysis, LS significantly correlated with the fibrosis stage $(r=0.70, P=$ $0.000)$, moderately correlated with the ALT value $(\mathrm{r}=0.398, \mathrm{P}=0.002)$, and poorly correlated with the degree of necroinflammation $(\mathrm{r}=0.19, \mathrm{P}=0.002)$. This is similar to the results of 
previous studies (Foucher et al., 2006; Ferraioli et al., 2013). LS was poorly but negatively correlated with serum albumin levels. Although LS was moderately correlated with the platelet count, there was no statistical significance $(r=-0.05, P=0.715)$. This result is in contrast to that of previous studies. The main reasons for this again could have been differences in the CLD etiology. The current study excluded patients with hepatic decompensation, which often has significant effects on the platelet count. Multivariate regression analysis showed that LS measurements correlated only with the fibrosis stage $(\mathrm{P}=0.000)$ and with no other variables.

The results of S0-4 by LB compared with those of LS determined using the standard values provided by the manufacture for F0-4 were very different. The primary reason for this may be the small number of cases.

In conclusion, LS measurement is a good method for the diagnosis of severe fibrosis and compensated cirrhosis. However, the shear wave values for mild, moderate, and severe fibrosis are not consistently accurate. Specific LS measurement standards for F0-4 based on various causes of CLD may improve the accuracy. Further investigation with larger sample sizes will be necessary to confirm these results.

\section{ACKNOWLEDGMENTS}

Research not supported by a specific grant.

\section{REFERENCES}

Arena U, Vizzutti F, Abraldes JG, Corti G, et al. (2008). Reliability of transient elastography for the diagnosis of advanced fibrosis in chronic hepatitis C. Gut 57: 1288-1293.

Calvaruso V, Di Marco V, Ferraro D, Petta S, et al. (2013). Fibrosis evaluation by transient elastography in patients with long-term sustained HCV clearance. Hepat. Mon. 13: e7176.

Carrión JA, Navasa M, Bosch J, Bruquera M, et al. (2006). Transient elastography for diagnosis of advanced fibrosis and portal hypertension in patients with hepatitis $\mathrm{C}$ recurrence after liver transplantation. Liver Transpl. 12: 1791-1798.

Castéra L (2008). Non-invasive diagnosis of steatosis and fibrosis. Diabetes Metab. 34 (6 Pt 2): 674-679.

Castéra L (2011). Non-invasive assessment of liver fibrosis in chronic hepatitis C. Hepatol. Int. 5: 625-634.

Castéra L, Vergniol J, Foucher J, Le Bail B, et al. (2005). Prospective comparison of transient elastography, Fibrotest, APRI, and liver biopsy for the assessment of fibrosis in chronic hepatitis C. Gastroenterology 128: 343-350.

Castéra L, Forns X and Alberti A (2008). Non-invasive evaluation of liver fibrosis using transient elastography. J. Hepatol. 48: 835-847.

Chen YP, Liang XE, Dai L, Zhang Q, et al. (2012). Improving transient elastography performance for detecting hepatitis B cirrhosis. Dig. Liver Dis. 44: 61-66.

de Ledinghen V and Vergniol J (2008). Transient elastography (FibroScan). Gastroenterol. Clin. Biol. 32: 58-67.

de Lédinghen V, Douvin C, Kettaneh A, Ziol M, et al. (2006). Diagnosis of hepatic fibrosis and cirrhosis by transient elastography in HIV/hepatitis C virus-coinfected patients. J. Acquir. Immune Defic. Syndr. 41: 175-179.

de Lédinghen V, Vergniol J, Barthe C, Foucher J, et al. (2013). Non-invasive tests for fibrosis and liver stiffness predict 5-year survival of patients chronically infected with hepatitis B virus. Aliment. Pharmacol. Ther. 37: 979-988.

Feier D, Lupsor Platon M, Stefanescu H and Badea R (2013). Transient elastography for the detection of hepatocellular carcinoma in viral C liver cirrhosis. Is there something else than increased liver stiffness? J. Gastrointestin. Liver Dis. 22: 283-289.

Ferraioli G, Tinelli C, Dal Bello B, Zichhetti M, et al. (2013). Performance of liver stiffness measurements by transient elastography in chronic hepatitis. World J. Gastroenterol. 19: 49-56.

Foucher J, Chanteloup E, Vergniol J, Castera L, et al. (2006). Diagnosis of cirrhosis by transient elastography (Fibro Scan): a prospective study. Gut 55: 403-408.

Fraquelli M, Rigamonti C, Casazza G, Conte D, et al. (2007). Reproducibility of transient elastography in the evaluation of liver fibrosis in patients with chronic liver disease. Gut 56: 968-973.

Fraquelli M, Rigamonti C, Casazza G, Donato MF, et al. (2011). Etiology-related determinants of liver stiffness values in chronic viral hepatitis B or C. J. Hepatol. 54: 621-628. 
Ganne-Carrié N, Ziol M, de Ledinghen V, Douvin C, et al. (2006). Accuracy of liver stiffness measurement for the diagnosis of cirrhosis in patients with chronic liver diseases. Hepatology 44: 1511-1517.

Kazemi F, Kettaneh A, N'kontchou G, Pinto E, et al. (2006). Liver stiffness measurement selects patients with cirrhosis at risk of bearing large oesophageal varices. J. Hepatol. 45: 230-235.

Lemoine M, Katsahian S, Ziol M, Nahon P, et al. (2008). Liver stiffness measurement as a predictive tool of clinically significant portal hypertension in patients with compensated hepatitis $\mathrm{C}$ virus or alcohol-related cirrhosis. Aliment. Pharmacol. Ther. 28: 1102-1110.

Rigamonti C, Donato MF, Fraquelli M, Agnelli F, et al. (2008). Transient elastography predicts fibrosis progression in patients with recurrent hepatitis C after liver transplantation. Gut 57: 821-827.

Rockey DC (2008). Noninvasive assessment of liver fibrosis and portal hypertension with transient elastography. Gastroenterology 134: 8-14

Sandrin L, Fourquet B, Hasquenoph JM, Yon S, et al. (2003). Transient elastography: a new noninvasive method for assessment of hepatic fibrosis. Ultrasound Med. Biol. 29: 1705-1713.

Shaheen AA, Wan AF and Myers RP (2007). FibroTest and FibroScan for the prediction of hepatitis C-related fibrosis: a systematic review of diagnostic test accuracy. Am. J. Gastroenterol. 102: 2589-2600.

Stebbing J, Farouk L, Panos G, Anderson M, et al. (2010). A meta-analysis of transient elastography for the detection of hepatic fibrosis. J. Clin. Gastroenterol. 44: 214-219.

Takeda T, Yasuda T, Nakayama Y, Nakaya M, et al. (2006). Usefulness of noninvasive transient elastography for assessment of liver fibrosis stage in chronic hepatitis C. World J. Gastroenterol. 12: 7768-7773.

Tsochatzis EA, Gurusamy KS, Ntaoula S, Cholongitas E, et al. (2011). Elastography for the diagnosis of severity of fibrosis in chronic liver disease: a meta-analysis of diagnostic accuracy. J. Hepatol. 54: 650-659.

Vizzutti F, Arena U, Romanelli RG, Rega L, et al. (2007). Liver stiffness measurement predicts severe portal hypertension in patients with HCV-related cirrhosis. Hepatology 45: 1290-1297.

Ziol M, Handra-Luca A, Kettaneh A, Christidis C, et al. (2005). Noninvasive assessment of liver fibrosis by measurement of stiffness in patients with chronic hepatitis C. Hepatology 41: 48-54. 